\section{$\underset{\substack{\text { hommes } \\ \text { \& migrations }}}{ }$}

\section{Hommes \& migrations}

Revue française de référence sur les dynamiques

migratoires

\section{$1279 \mid 2009$}

L'Afrique en mouvement

\title{
Les Mozambicains de Johannesburg
}

Nouveaux aspects d'une migration intra-africaine ancienne

\section{Dominique Vidal}

\section{(2) OpenEdition}

\section{Journals}

Édition électronique

URL : http://journals.openedition.org/hommesmigrations/338

DOI : $10.4000 /$ hommesmigrations.338

ISSN : 2262-3353

Éditeur

Musée national de l'histoire de l'immigration

\section{Édition imprimée}

Date de publication : 1 mai 2009

Pagination : 114-125

ISSN : 1142-852X

\section{Référence électronique}

Dominique Vidal, « Les Mozambicains de Johannesburg», Hommes \& migrations [En ligne], 1279|

2009, mis en ligne le 29 mai 2013, consulté le 14 novembre 2019. URL : http://

journals.openedition.org/hommesmigrations/338; DOI : 10.4000/hommesmigrations.338 


\section{Les Mozambicains de Johannesburg \\ Nouveaux aspects d'une migration intra-africaine ancienne}

Par Dominique Vidal, université Paris Diderot - Paris 7/URMIS

Capitale de la province du Gauteng, la plus riche d'Afrique du Sud, Johannesburg attire de nombreux migrants. Depuis plus d'un siècle, les Mozambicains s'y rendent pour trouver du travail et faire vivre leur famille. Alors qu'ils fuient un pays dévasté par la guerre, la fin de l'apartheid les place en concurrence directe avec les Sud-Africains. Entre les difficultés du retour et la xénophobie ambiante, les migrants du Mozambique tentent de se fondre dans la métropole. Décryptage d'une migration en milieu hostile. 
L'adaptation des migrants à la ville a été l'un des thèmes majeurs de l'anthropologie sociale en Afrique australe de la fin des années cinquante jusqu'aux années quatre-vingt ${ }^{(1)}$. Les migrations vers les villes ont augmenté depuis, mais les études urbaines les ont délaissées pour s'intéresser à la ville africaine comme lieu de créativité et de syncrétisme ${ }^{(2)}$. Si des travailleurs font le voyage du Mozambique à Johannesburg depuis la fin du $\mathrm{XX}^{\mathrm{e}}$ siècle, la nature de cette migration a considérablement évolué autour du milieu des années quatre-vingt ${ }^{(3)}$. Avec la fin des limites imposées aux déplacements des Africains du temps de l'apartheid ${ }^{(4)}$, la ville est devenue le centre de nombreux flux migratoires intérieurs et internationaux. Quel sort est réservé aux migrants dans la métropole sud-africaine ? Une enquête sur les ressortissants du Mozambique permet d'y répondre ${ }^{(5)}$.

\section{Une migration séculaire structurée}

Selon les plus anciens témoignages, les conditions topographiques et climatologiques ingrates du sud du Mozambique - en particulier dans ce qui est aujourd'hui la province de Gaza - ont poussé les habitants des zones rurales à quitter le sol de la colonie portugaise ${ }^{(6)}$ dès - au moins - le XIX ${ }^{\text {e }}$ siècle. Ces mobilités ne rencontrent pas alors d'obstacle de l'État portugais qui n'exercera jamais qu'une faible emprise sur le Mozambique. Lisbonne ne cherche véritablement à le coloniser que dans les années trente quand le général Salazar encourage l'installation de colons. À la fin des années dix-huit cent cinquante, des hommes arrivent par le port de Durban pour travailler dans les plantations sucrières de la colonie britannique du Natal, afin de pallier l'insuffisance de la main-d'ceuvre venue des Indes et la défiance des colons à l'égard des Zoulous. Les débuts de l'exploitation de gisements diamantifères à Kimberley, dans l'État libre d'Orange, en 1867, entraînent un accroissement des migrations du sud du Mozambique vers cette région faiblement peuplée du centre de l'Afrique du Sud.

En 1886, la découverte de filons d'or sur le site de ce qui devient la même année la ville de Johannesburg intensifie ce flux. La mobilité des travailleurs entre les deux territoires se déroule dans le cadre qui a reçu le nom, en Afrique australe, de "système du travail migrant" (Migrant Labour System). Ce dispositif se met progressivement en place après l'ouverture des premières mines d'or et s'étend par la suite à tout le secteur minier et à une partie de l'agriculture. Il a pour caractéristique fondamentale d'organiser la migration temporaire, pour des périodes de douze à dix-huit mois, d'hommes des zones rurales de l'Afrique du Sud et des pays avoisinants. Ces migrants ne perçoivent sur place qu'une faible part de leur 
rémunération et touchent le principal seulement de retour au pays. Ils se réinstallent alors dans leur village en attendant d'être éventuellement rappelés.

\section{Le travail migrant ou l'instrument de l'exil}

La rotation de ces travailleurs poursuit un double objectif. Elle entend, d'une part, interdire la formation d'une classe ouvrière, un objectif renforcé par la mise en concurrence des différents groupes ethniques dans les mines. Elle vise, d'autre part, à empêcher l'urbanisation des Africains, tenus comme irréductiblement ruraux et n'ayant pour seule raison de se trouver dans les zones industrielles que la satisfaction des besoins en main-d'ceuvre des Blancs. Institué en 1948, dans une période de forte industrialisation, l'apartheid développe ce système de travail migrant, en s'appuyant sur les Africains des régions limitrophes de l'Afrique du Sud, afin de ne pas accentuer la migration des campagnes vers les villes sur son propre sol.

De toute l'Afrique australe, le Mozambique fournit le plus d'hommes au système du travail migrant, dès ses premiers temps. Une série d'accords entre le Portugal et la République du Transvaal d'abord, puis avec le dominion britannique de l'Union sud-africaine après 1910, conduit à l'organisation d'une migration de travail entre le sud du Mozambique et la région du Witwatersrand. Modeste port jusqu'en 1887, Lourenço Marques (Maputo depuis l'indépendance en 1975) devient la ville la plus riche de la colonie et accède, en 1898, au statut de capitale à la place de l'île de Mozambique, à l'extrême nord du pays. Au début du XX $\mathrm{XX}^{\mathrm{e}}$ siècle, la Chambre des mines obtient des Portugais le droit d'installer des bureaux de recrutement au sud du fleuve Save, qui coupe le Mozambique le long du vingtdeuxième parallèle. En contrepartie de sa coopération à l'encadrement de ce flux, le Portugal se voit garantir par l'Afrique du Sud un transit important par le port de Lourenço Marques et l'acquisition d'or à un prix inférieur à celui du marché international.

Le système du travail migrant modifie en profondeur les formes d'organisation sociale dans le sud du Mozambique. Il produit, en premier lieu, un puissant imaginaire de la migration dans ces régions où, entre 1902 et 1977, il concerne entre $25 \%$ et $30 \%$ des hommes en âge de partir vers les mines. L'absence et l'argent de ces derniers entraînent, en deuxième lieu, un faible développement de l'agriculture qui se limite essentiellement à l'autosuffisance des familles. Rares sont en effet les unités familiales du sud du fleuve Save qui n'ont pas bénéficié des gains des mineurs ${ }^{(7)}$. Le statut social qu'ils acquièrent affaiblit, enfin, l'autorité traditionnelle des chefs et des aînés, tant la capacité d'importer du bétail et des biens venus 
d'Afrique du Sud assure du prestige jusqu'au plus profond des zones rurales ${ }^{(8)}$. L'imbrication du sud du Mozambique dans l'économie sud-africaine et sa dépendance économique n'ont jamais cessé depuis, même au plus fort des tensions entre Pretoria et Maputo quand les dirigeants du Frelimo revendiquaient leur appartenance au camp socialiste ${ }^{(9)}$.

La nature des migrations du Mozambique vers l'Afrique du Sud change considérablement à partir des années quatre-vingt avec, dans le premier pays, l'intensification de la guerre civile puis l'accord de paix entre les belligérants en 1992, et, dans le second, l'élection de Nelson Mandela à la présidence de la République en 1994.

\section{Le tournant des années quatre-vingt}

Si, pendant cette période, le système du travail migrant continue de structurer le séjour en Afrique du Sud de Mozambicains, la plupart franchissent la frontière pour de toutes autres raisons que le travail dans les mines. Les violences et la misère que provoque le conflit entre le Front de libération du Mozambique (Frelimo) au pouvoir et la Résistance nationale du Mozambique (Renamo) poussent quelque 
350000 Mozambicains à fuir leur pays pour trouver refuge chez leur voisin du Sud. La majorité s'installe dans les zones rurales de la région du Limpopo, de l'autre côté du parc du Kruger, et notamment dans les villages où vivent des Sud-Africains du même groupe ethnique shangaan. Pretoria les tolère tant qu'ils ne quittent pas ces ter-

Les revenus obtenus des emplois les plus mal rémunérés dans la métropole sud-africaine sont en effet toujours bien plus élevés que ceux qu'ils peuvent espérer au Mozambique. ritoires situés, du temps de l'apartheid, dans les bantoustans du Gazankulu et du KaNgwane, mais sa police les reconduit à la frontière dès qu'elle les arrêtent dans les zones urbaines ou à leur proximitér ${ }^{100}$. Cela n'empêche pas des dizaines de milliers de Mozambicains de s'installer à Johannesburg à partir de la seconde moitié

des années quatre-vingt. La plupart de ces migrants viennent de la région de Maputo où sévit une pauvreté aggravée par la croissance exponentielle de l'agglomération due aux migrations intérieures depuis le déclenchement de la guerre civile. Ce sont pour l'essentiel de jeunes hommes le plus souvent autour de dix-huit ans. Certains cherchent à échapper à la conscription dans un bataillon aux confins du pays. Mais la motivation principale du plus grand nombre reste la même que celle qui animait leurs aînés qui allaient travailler dans les mines. Ils partent dans l'espoir de revenir un jour au pays forts d'une épargne suffisante pour accéder à la propriété, ouvrir un commerce et se trouver en position avantageuse sur le marché matrimonial.

\section{Subsister à Jo'Burg : le règne de l'informel}

Mais que font au juste ces migrants à Johannesburg ? Le secteur minier n'en compte plus qu'environ soixante mille. Sa modernisation a conduit à la réduction des effectifs et les pressions des syndicats sud-africains se sont traduites par une politique d'emploi privilégiant les nationaux ${ }^{(11)}$. Les autres, hormis une petite minorité de techniciens et de cadres, travaillent dans le secteur informel de l'économie. On les trouve en particulier dans le bâtiment, la restauration, la manutention, la carrosserie et les emplois de rue (coiffeurs, vendeurs sur les trottoirs) ${ }^{(12)}$. Leur forte présence dans ces secteurs résulte non seulement de la faible qualification de la plupart d'entre eux, mais aussi du différentiel considérable des rémunérations entre Maputo et Johannesburg. Les revenus obtenus des emplois les plus mal rémunérés dans la métropole sud-africaine sont en effet toujours bien plus élevés que ceux qu'ils peuvent espérer au Mozambique. Ils suffisent souvent à la 
constitution de la petite épargne nécessaire à la réalisation du projet migratoire. L'African Cities Project a d'ailleurs mis en évidence le très fort taux d'activité des Mozambicains : la quasi-totalité travaillent à Johannesburg, dont plus de la moitié à temps complet, des chiffres de très loin plus élevés que ceux des Noirs sudafricains, des Congolais et des Somaliens, beaucoup moins souvent employés à plein temps et connaissant un taux de chômage oscillant entre $40 \%$ et $20 \%$. Cette enquête pionnière établit en outre que, de ces quatre groupes, les migrants mozambicains ont en moyenne les revenus les plus élevés, même s’ils restent modestes. Or les Noirs sud-africains les méprisent pour cette concurrence qu'ils jugent déloyale, tout en concédant que ce sont de gros travailleurs.

\section{Des victimes de la xénophobie}

Ce dernier point amène à évoquer la xénophobie dont pâtissent les migrants mozambicains, comme tous les autres étrangers africains. Elle est une dimension essentielle de leur expérience et se traduit par des comportements qui vont de l'insulte verbale à l'assassinat ${ }^{(13)}$. Cette xénophobie ne peut être comprise sans que soit évoqué ce que fut l'apartheid, et ses conséquences ${ }^{(14)}$.

Cette politique avait, on le sait, pour objectif d'assurer la suprématie raciale des individus classés dans la catégorie "Whites" (ou Europeans). Elle conduit à assigner des lieux de résidence à la population africaine, à la périphérie des villes ou dans les zones rurales, et retire graduellement à ses membres la nationalité sudafricaine. Après 1994, l'instauration d'un régime démocratique en Afrique du Sud modifie en profondeur le tracé des frontières spatiales et juridiques qui organisaient les déplacements et séparaient le national de l'étranger ${ }^{(15)}$. Si, du temps de l'apartheid, seuls les Blancs possédaient une citoyenneté pleine, tous les SudAfricains se voient maintenant reconnus en droit. En conséquence, la "race" n'est plus ce qui distingue le citoyen du non-citoyen. Il résulte de ce changement radical de conception du national l'établissement d'une frontière entre les Africains de nationalité sud-africaine et ceux originaires d'autres pays du continent, une frontière qui contribue à la formation d'une nouvelle forme d'appartenance alimentant la xénophobie ordinaire des premiers à l'égard des seconds.

Les gouvernements de l'Afrique du Sud post-apartheid n'apportent pas de réponses à ces comportements entretenus par des médias qui relaient les représentations dominantes de ces nouveaux venus, supposés vecteurs du chômage, du sida et de la criminalité. La police, également xénophobe et notoirement corrompue, rackette de son côté les clandestins plus qu'elle ne protège les migrants des agressions. 


\section{Les Mozambicains en première ligne}

Des mesures de régularisation sont bien adoptées dans la seconde moitié des années quatre-vingt-dix, mais elles ne concernent que peu d'entre eux dans une période où se met en place une politique migratoire restrictive qui entraîne des reconduites à la frontière d'une ampleur sans précédent. Dans les dix années qui suivent l'élection de Nelson Mandela à la présidence de la République sud-africaine en 1994, on compte 1.15 millions de Mozambicains parmi les 1.5 millions d'étrangers expulsés. En 2005, un accord est signé entre le Mozambique et l'Afrique du Sud, permettant aux ressortissants des deux pays d'obtenir facilement un visa d'une durée maximale de trente jours pour séjourner dans l'État voisin. Il a facilité la vie de beaucoup de migrants mozambicains qui franchissent la frontière pour acheter des biens qu'ils revendent de l'autre côté, effectuer des missions de travail de courte durée ou rendre visite à des parents. Il n'a pas pour autant mis fin à la présence massive de sans-papiers mozambicains en Afrique du Sud ni à la xénophobie qu'ils rencontrent à Johannesburg.

La dureté de la vie dans la métropole sud-africaine ne dissuade pas les jeunes hommes de Maputo d'en prendre le chemin, afin d'accumuler le capital nécessaire à l'achat d'une habitation et à l'ouverture d'un commerce dans leur pays. De la sorte, ils espèrent échapper à cette double situation de dépendance que représente le fait de devoir résider au domicile familial, où ils subissent la tutelle paternelle, et de travailler pour un employeur les exploitant sans vergogne.

\section{Un autre type d'"aventurier"?}

La littérature sur les migrations en Afrique subsaharienne insiste sur l'importance de la figure sociale de 1'“aventurier", tant dans l'autoreprésentation des jeunes migrants que dans les discours sur ces derniers tenus par des locuteurs aussi divers que des populations plus anciennement installées dans les pays de transit, les responsables politiques, les journalistes, les organisations non-gouvernementales et les chercheurs eux-mêmes ${ }^{(16)}$.

On retrouve comme tels ces termes d'"aventure" (aventura) et d'"aventurier" (aventureiro) chez les migrants et anciens migrants mozambicains. Ils possèdent une histoire que Sylvie Bredeloup a relevée en Afrique de l'Ouest et au Congo ${ }^{(17)}$. De vieux mineurs de plus de soixante-dix ans rencontrés à Maputo se souvenaient ainsi de leurs "aventures" et parlaient de leurs pères et de leurs grandspères qui les avaient précédés en Afrique du Sud comme "aventuriers". La migra- 
tion en Afrique du Sud, comme étape nécessaire à l'entrée dans un statut adulte, est du reste un thème qui traverse les études sur les migrations du sud du Mozambique vers son riche voisin ${ }^{(18)}$.

Faut-il pour autant affirmer la continuité des migrations mozambicaines vers Johannesburg sur la base de cette continuité des représentations du projet migratoire exprimées par le vocabulaire de l'aventure? Morten Madsen s'engage dans cette voie dans son étude sur un groupe de jeunes hommes originaires de Maputo à Johannesburg ${ }^{(19)}$. Il analyse ainsi la migration clandestine comme un rite de passage, dans un sens voisin de celui qu'Arnold Van Gennep donne à ce terme et en lui empruntant, notamment, son idée moins connue de "passage territorial". Pour Madsen, partir pour l'Afrique du Sud représente pour ces migrants une phase liminaire au terme de laquelle ils peuvent espérer une nouvelle position au Mozambique. Il insiste toutefois sur le fait qu'à la différence des rites de passage décrits par Van Gennep cette période liminaire n’a pas dans leur cas de terme prédéfini et peut ne jamais s'achever. Au point qu'il est peut-être plus approprié de parler de "transit prolongé" (Protracted Transience). 


\section{Une aventure ambiguë}

Mon enquête multisituée à Johannesburg et à Maputo amène cependant à considérablement nuancer, sans l'invalider pour autant, l'analyse de Madsen. Beaucoup de migrants mozambicains regagnent aujourd'hui le Mozambique les mains vides après avoir été volés ou reconduits à la frontière. Et ceux qui reviennent avec une épargne n'ont pas toujours assez d'argent pour se mettre à leur compte. Aussi, une approche de cette migration centrée sur le thème du rite de passage ne rend pas suffisamment compte des changements qui l'ont marquée depuis plus de deux décennies et des dynamiques à l'ceuvre en son sein. Car, on le sait, la migration peut s'étendre sur des décennies et devenir une condition sociale permanente.

Ces jeunes Mozambicains diffèrent des autres Africains du même âge qui se disent dans le même registre de l'aventure. L'individu qu'ils révèlent se détache sur fond d'une érosion plus ancienne des traditions. Les changements provoqués par le système du travail migrant depuis la fin du XIX ${ }^{\mathrm{e}}$ siècle ont eu pour conséquence, dans le sud du Mozambique, l'affirmation, dans les zones rurales puis en ville, de groupes de parenté de taille restreinte dont les membres ne sont pas astreints à des solidarités communautaires fortes, comme cela a été observé en Afrique de l'Ouest. Le migrant mozambicain dans le Johannesburg post-apartheid a pour cela un caractère plus dépouillé. Cette rusticité se repère notamment dans la difficulté à tenir un discours élaboré sur soi, la méfiance à l'égard d'autrui et la conviction que les meilleures réponses à l'adversité passent par la capacité à savoir maîtriser son environnement pour en tirer le meilleur parti.

\section{Tenir et se tenir dans une métropole hostile}

Les migrants mozambicains de Johannesburg ne cherchent ni à se regrouper ni à s'organiser sur la base du partage d'une identité ethnique ou nationale. Ils vivent certes souvent avec d'autres compatriotes, mais ils ne se concentrent dans aucun espace particulier de la ville, comme les Congolais, les Nigérians et les Somaliens. Ils n'ont également pour la grande majorité d'entre eux aucun contact avec les quelques associations ayant pour vocation, selon leurs dirigeants, de "représenter la communauté mozambicaine".

C'est en réalité la recherche de l'invisibilité qui caractérise ces Mozambicains. Celle-ci tient, d'une part, à l'absence d'espaces et de collectifs qui pourraient constituer un support facilitant leur vie à Johannesburg. Mais elle procède aussi, d'autre part, de la fragilité des liens sociaux et du manque de confiance de ces migrants 
envers autrui. L'enquête ethnographique comme les statistiques de l'ACP ont montré qu'ils ne peuvent souvent pas compter durablement sur le soutien de parents et de compatriotes à Johannesburg.

\section{L'individu comme stratégie}

Beaucoup d'entre eux préfèrent en outre s'engager dans des stratégies individuelles plutôt que de construire une position stable en s'organisant pour défendre collectivement leurs intérêts. La logique de ces comportements - dans lesquels il est bien difficile de voir une stratégie au sens fort tant ils sont plus imposés que délibérément choisis - résulte en partie de la proximité géographique du Mozambique qui leur permet de revenir fréquemment dans leur pays d'origine, notamment lorsqu'ils traversent une mauvaise passe. Elle découle aussi de leur préférence généralisée pour une migration temporaire, même si, dans les faits, nombre d'entre eux ne mettent jamais fin à leur vie de migrant ${ }^{(20)}$. Le regroupement familial n'est ainsi pas un objectif des Mozambicains, mais une contrainte supplémentaire. Quand ils le peuvent, ils préfèrent que leur compagne reste au pays à élever les enfants ou, si elle les rejoint à Johannesburg, que les enfants grandissent au Mozambique au domicile des grands-parents.

La stabilisation de la position des migrants mozambicains s'opère essentiellement au moyen de la construction d'ententes à un échelon microlocal. L'enquête a ainsi établi que les principales difficultés qu'ils rencontrent (les agressions et les reconduites à la frontière) se produisent au cours des deux premières années de leur présence sur le territoire sud-africain. Durant ces premiers temps, ils ne maîtrisent pas encore suffisamment les façons de parler, de s'habiller et de se comporter qui permettent de se fondre dans l'univers urbain.

\section{Des migrants invisibles}

Une fois inscrits dans des échanges qui reposent sur l'interconnaissance et des pratiques d'entraide, les Mozambicains disent ne pas rencontrer plus de difficultés que les Sud-Africains. Ils n'échappent pas pour autant aux propos xénophobes que beaucoup de ces derniers ont vite à la bouche, mais ces incidents, pour désagréables et déstabilisants qu'ils soient, restent le plus souvent sans autre conséquence là où les migrants sont favorablement connus. Venus pour l'essentiel de zones urbaines, ils font dans la métropole sud-africaine une autre expérience de la 
condition de citadin. Celle-ci diffère non seulement de celle qu'ils faisaient dans une ville pauvre, dégradée et au rythme plus lent comme Maputo, mais elle n'a guère à voir également avec celle de l'habitant des villes décrit par un Simmel ou un Wirth. Pour tenir à Johannesburg, il leur faut par conséquent, on le voit, à la fois savoir passer inaperçus dans les espaces où personne ne les connaît et parvenir à faire oublier la différence qui stigmatise, en se faisant reconnaître comme des partenaires d'interaction de qualité dans les lieux où ils résident et travaillent. À la différence de ce qui a pu être observé dans le Copperbelt zambien et en Afrique de l'Ouest, ils sont majoritairement animés par une confiance en l'avenir. Il y aurait là tout du paradoxe si l'on ne considérait que la dureté de leur existence en Afrique du Sud. Mais ce serait négliger que cet optimisme n'est intelligible que rapporté à la misère et à la dévastation qu'a connues le Mozambique. Et si cela n'en fait assurément pas des migrants satisfaits de leur sort, cette représentation prévalant parmi eux rappelle combien l'évaluation de l'expérience migratoire par le migrant est fonction du jugement qu'il porte sur le monde qu'il quitte, où il espère revenir en vainqueur.

\section{Notes}

1. Voir en particulier : James Clyde Mitchell, "La danse du Kalela", (1956), Enquête n ${ }^{\circ} 4,1996$, p.213-243 ; Max Gluckman, "Analysis of a Social Situation in Modern Zululand", Rhodes Livingstone Paper n²8, 1958, Manchester, Manchester University Press ; Arnold Leonard Epstein, Politics in an Urban African Community, Manchester, Manchester University Press, 1958 ; Philip Mayer, Townsmen or Tribesmen. Conservatism and the Process of Urbanization in a South African City, (1961), Cape Town, Oxford University Press, 1971.

2. Catherine Coquery-Vidrovitch, "De la ville en Afrique noire", Annales HSS, sept.-oct., n5, 2006, p.1087-1119.

3. Il n'existe ici encore aucune donnée précise sur le nombre de Mozambicains ou de Sud-africains d'origine mozambicaine en Afrique du Sud. Les chiffres circulant à ce propos varient entre trois cent mille et plus de deux millions.

4. Il est toutefois impossible, comme souvent en matière de connaissance des migrations, de fournir un chiffre un tant soit peu précis sur le nombre de ces nouveaux migrants, une majorité d'entre eux allant et venant entre Johannesburg et les zones rurales dont ils sont originaires ou étant des étrangers dans la ville en situation de clandestinité.

5. Cette enquête a bénéficié du soutien de l'Institut français d'Afrique du Sud (IFAS) et s'appuie sur des matériaux recueillis au cours de quatre missions d'une durée totale de sept mois. Ceux-ci consistent en des entretiens de migrants et anciens migrants de sexe masculin, l'observation de différents sites, ainsi que des données statistiques issues de l'African Cities Project (ACP) de 2006, une étude longitudinale et comparative des réfugiés, des demandeurs d'asile, des migrants et des non nationaux dans les quartiers centraux (inner cities) de Johannesburg, Lubumbashi (République démocratique du Congo), Maputo (Mozambique) et Nairobi (Kenya). Si l'ACP a produit des données sur les migrants des deux sexes, mes enquêtes ne portent que sur les hommes, lesquels constituent l'essentiel de ce courant migratoire. La gêne des Mozambicaines à être vues en présence d'un Européen blanc, une situation potentiellement déchiffrable comme l'indice d'une activité prostitutionnelle, m'a en effet amené à renoncer à les prendre pour objet pour éviter d'accroître leur vulnérabilité déjà grande.

6. Isak. Niehaus, "Ethnicity and the Boundaries of Belonging: Reconfiguring Shangaan Identity in the South African lowveld", African Affairs, 101, 2002, p.557-583.

7. Avec un taux de masculinité inférieur à 80 hommes pour 100 femmes, accentué en particulier dans la tranche entre 15 et 55 ans, cette région du Mozambique possède aujourd'hui une caractéristique démographique qui la différencie nettement du reste de la population. Voir Michel Cahen, Philippe Waniez, Violette Brustlein, "Pour un atlas social et culturel du Mozambique", Lusotopie n¹, 2002, p.365-362. 
8. Voir Patrick Harries, Work, Culture, and Identity. Migrant Laborers in Mozambique and South Africa, 1860-1910, Johannesburg, Witwatersrand University Press, 1994.

9. Michel Cahen, Mozambique. La révolution implosée : études sur douze ans d'indépendance (1975-1987), Paris, L'Harmattan, 1987.

10. Aurelia Wa Kabwe-Segatti, "Du rapatriement volontaire au refoulement dissimulé. Les réfugiés mozambicains en Afrique du Sud", Politique africaine n ${ }^{\circ} 85,2002$, p.75-92.

11. Il n'en reste pas moins toutefois que le nombre de mineurs mozambicains dans les mines sud-africaines reste toujours plus important que le nombre total de salariés du secteur manufacturier au Mozambique (Wa Kabwe-Segatti, 2009).

12. Je n'évoque pas ici les activités illégales (celles notamment liées à la contrebande et au trafic de biens volés vers le Mozambique). Bien que les Mozambicains aient eu une solide réputation de criminels en Afrique du Sud, les statistiques sur la population pénale du pays n'indiquent pas qu'ils y soient surreprésentés, sans qu'il soit possible d'être assuré que cela découle du caractère infondé de cette réputation ou à leur discrétion en la matière.

13. En quatre semaines, les exactions à l'encontre des étrangers ont fait une trentaine de victimes dans la région de Johannesburg et entraîné le retour dans leur pays d'origine - définitif ou momentané, nul ne le sait encore de manière précise - de près de 36000 Mozambicains.

14. Aurelia Wa Kabwe-Segatti, '“Clandestins' et 'Makwerekwere' dans l'Afrique du Sud post-apartheid : production de catégories, pratiques administratives et xénophobie", Information sur les sciences sociales, vol. 47 (4), 2008, p.661-680.

15. Dominique Vidal, "Vivre sur fond de frontières. Les migrants du Mozambique à Johannesburg", Cultures\&Conflits n²72, 2008, p.101-117.

16. Philippe Poutignat, Jocelyne Streiff-Fenart, "Nouadhibou 'ville transit' ? Le rapport d'une ville à ses étrangers dans le contexte des politiques de contrôle des frontières de l'Europe", Revue européenne des migrations internationales, 24 (2), 2008, p.193-217 ; Mahamet Timera, (sous presse). "'Aventuriers' ou orphelins de la migration transnationale ? 'Nouveaux venus' et 'anciens' migrants 'subsahariens' au Maroc", Politique africaine, 2009.

17. Sylvie Bredeloup, "L'aventurier, une figure de la migration africaine", Cahiers internationaux de sociologie, $\mathrm{n}^{\circ} 2$, 2008, p. 281-306

18. Voir notamment l'enquête dirigée par l'anthropologue sud-africaine Ruth First dans les zones rurales de la province d'Inhambane, O mineiro moçambicano. Um estudo sobre a exportaçáo de máo de obra em Inhambane, Maputo, Universidade Eduardo Mondlane, Centro de estudos africanos, 1998 (1977).

19. Morten Madsen, A Walk through Thick Bushes. An Ethnographic Account of Undocumented Mozambicans in Johannesburg, Master's Thesis, University of Copenhagen, 2004.

20. Cela n'est cependant pas spécifique aux Mozambicains, mais constitue, comme le souligne Victor Agadjnian, une des caractéristiques des migrants d'Afrique australe en Afrique du Sud, caractéristique résultant largement des formes de circulation et de rotation mises en place du temps où prévalait le système du travail migrant. Victor Agadjnian, "Research on International Migration within Sub-Saharan Africa: Foci, Approaches, and Challenges", The Sociological Quaterly, vol. 49, n³, 2008, p.407-421.

\section{Bibliographie}

- Jonathan Crush, Alan Jeeves, David Yudelman, South Africa's Labor Empire. A History of Black Migrancy to the Gold Mines, Cape Town, David Philip, 1991.

- Christophe Daum, "La coopération, alibi de l'exclusion des immigrés? L'exemple malien", in Didier Fassin, Alain Morice et Catherine Quiminal (eds.), Les lois de l'hospitalité. Les politiques de l'immigration à l'épreuve des sans-papiers, Paris, La Découverte, 1997, p.197-216.

- James Ferguson, Expectations of Modernity. Myths and Meanings of Urban Life on the Zambian Copperbelt, Berkeley, University of California Press, 1999.

- Simon E. Katzenellenbogen, South Africa and Southern Mozambique. Labour, Railways and Trade in the Making of a Relationship, Manchester, Manchester University Press, 1982.

- Brigitte Lachartre, Enjeux urbains au Mozambique. De Lourenço Marques à Maputo, Paris, Karthala, 2000.

- Alain Marie, (ed.), L'Afrique des individus. Itinéraires citadins dans l'Afrique contemporaine (Abidjan, Bamako, Dakar, Niamey), Paris, Karthala, 1997.

- Alain Marie, François Leimdorfer (eds.), L'Afrique des citadins. Sociétés civiles en chantier (Abidjan, Dakar), Paris, Karthala, 2003.

- Deborah Posel, The Making of Apartheid, 1948-1961. Conflict and Compromise, Oxford, Clarendon Press, 1991.

- Abdelmalek Sayad, La double absence. Des illusions de l'émigré aux souffrances de limmigré, Paris, Éditions du Seuil, 1999.

- Aurelia Wa Kabwe-Segatti, (sous presse), "Les oubliés de la croissance mozambicaine : les migrants mozambicains dans l'Afrique du Sud de Mbeki", Lusotopie, XVI (1), 2009. 